\title{
Chiral 3NF and neutron-deuteron scattering
}

\author{
Henryk Witała*, Jacek Golak, Roman Skibiński ${ }^{\dagger}$ \\ M. Smoluchowski Institute of Physics, Jagiellonian University PL-30059 Kraków, Poland \\ E-mail: witala@if.uj.edu.pl, ufgolak@cyf-kr.edu.pl, \\ roman.skibinski@uj.edu.pl
}

Walter Glöckle

Institut für Theoretische Physik II, Ruhr-Universität Bochum, D-44780 Bochum, Germany

E-mail: Walter.Gloeckleetp2.ruhr-uni-bochum.de

\section{Hiroyuki Kamada}

Department of Physics, Faculty of Engineering, Kyushu Institute of Technology, 1-1 Sensuicho

Tobata, Kitakyushu 804-8550, Japan

E-mail: kamada@mns.kyutech.ac.jp

\section{Andreas Nogga}

Institut für Kernphysik and Jülich Center for Hadron Physics, Forschungszentrum Jülich,

D-52425 Jülich, Germany

E-mail: a.nogga@fz-juelich.de

Results on three-nucleon ( $3 \mathrm{~N})$ elastic scattering below the pion production threshold are discussed with emphasis on the need for a three-nucleon force (3NF). The large discrepancies found between a theory based on numerical solutions of $3 \mathrm{~N}$ Faddeev equations with (semi)phenomenological NN potentials only and data point to the need for 3NF's. This is further supported since another possible reason for the discrepancies in elastic nucleon-deuteron ( $\mathrm{Nd}$ ) scattering, relativistic effects, turned out to be small. Results for chiral NN (at $\mathrm{N}^{2} \mathrm{LO}$ and $\mathrm{N}^{3} \mathrm{LO}$ ) and $3 \mathrm{NF}^{\prime}$ 's (at $\mathrm{N}^{2} \mathrm{LO}$ ) are shown and discussed.

6th International Workshop on Chiral Dynamics

July 6-10 2009

Bern, Switzerland

\footnotetext{
*Speaker.

$\dagger$ This work was supported by the 2008-2011 polish science funds as the research project No. N N202 077435. It was also partially supported by the Helmholtz Association through funds provided to the virtual institute "Spin and strong QCD”(VH-VI-231). The numerical calculations were performed on the supercomputer cluster of the JSC, Jülich, Germany.
} 


\section{Introduction}

Traditionally in nuclear physics the Hamiltonian has been taken in a nonrelativistic form in which pairwise interactions between nucleons are supplemented by 3NF's for systems with more than two nucleons. The construction of NN potentials guided by a meson picture led to a generation of realistic $\mathrm{NN}$ interactions which describe the $\mathrm{NN}$ data set with high precision $\left(\chi^{2} /\right.$ datum $\left.\approx 1\right)$ [1], 2. 3]. The $3 \mathrm{~N}$ system is the first nontrivial case where those realistic potentials can be tested. In that system also the first time 3NF's come into play making it a valuable source of information on $3 \mathrm{NF}$ properties and their significance in the nuclear Hamiltonian.

The need for 3NF's was established when three- and four-nucleon bound states have been solved exactly using standard integration and differentiation methods [ techniques low energy states for nuclei up to $\mathrm{A}=12$ have been calculated [6]. It turned out that in all cases studied realistic NN forces alone provided clear underbinding, which for ${ }^{3} \mathrm{H}$ and ${ }^{3} \mathrm{He}$ amounts to $\approx 0.5-0.9 \mathrm{MeV}$ and for ${ }^{4} \mathrm{He}$ to $\approx 2-4 \mathrm{MeV}$.

It was natural to look for an explanation of this underbinding introducing $3 \mathrm{NF}$ 's in the nucleonic Hamiltonian. An important example for a process, which leads to a $3 \mathrm{NF}$, is a $\pi-\pi$ exchange between three nucleons with an intermediate $\Delta$ excitation of one nucleon, considered by Fujita-Miyazawa [7]. Later this process was incorporated into the Urbana IX 3NF, where it is supplemented by a phenomenological short-range spin- and isospin-independent part [8]. The Tucson-Melbourne (TM) model [9] was constructed using a more general $\pi \mathrm{N}$ amplitude. It also contains a strong form factor parametrization with a cut-off parameter $\Lambda$ which can be adjusted to the ${ }^{3} \mathrm{H}$ or ${ }^{3} \mathrm{He}$ binding energy when this $3 \mathrm{NF}$ is combined with a particular NN interaction. For the combination of the AV18 NN potential and the Urbana IX 3NF calculations are available for nuclei up to $A=12$ which show that one can reach a reasonable description of the low lying bound states in those nuclei [6]. However, the results show for instance an insufficient spin-orbit splitting and an insufficient charge dependence, which indicate defects of this $3 \mathrm{NF}$. Taking additional $3 \mathrm{NF}$ ring-diagrams into account the situation could be improved [6].

Recently application of effective field theoretical methods in a form of chiral perturbation theory $(\chi \mathrm{PT})$ provided a solid basis for construction of nuclear forces [10, 11]. Consistent chiral $\mathrm{NN}$ and $3 \mathrm{~N}$ forces have been derived in the $\chi \mathrm{PT}$ framework. They can also resolve the underbinding problems of ${ }^{3} \mathrm{H},{ }^{3} \mathrm{He}$ and ${ }^{4} \mathrm{He}$ nuclei [12] and lead to an improved description of p-shell nuclei properties [13].

A rich set of spin observables for elastic Nd scattering is a source of valuable information on the spin and momentum structure of 3NF's. In the next section we briefly review the $3 \mathrm{~N}$ scattering formalism and give some examples where data are compared to various NN potential predictions alone or combined with different 3NF's. These examples are chosen to show the importance of the $3 \mathrm{NF}$ in the $3 \mathrm{~N}$ system. The importance of $3 \mathrm{NF}$ 's seems to increase with increasing energy of the $3 \mathrm{~N}$ system. Therefore we discuss the importance of relativistic effects in that reaction and their significance in the study of $3 \mathrm{NF}$ effects. The smallness of relativistic effects, however, indicates that the focus is on 3NF's, specifically of short-range. Thus in the following section we present examples of results for chiral $\mathrm{NN}$ potentials alone and combined with $\mathrm{N}^{2} \mathrm{LO}$ chiral $3 \mathrm{~N}$ interactions, which, in addition to the $2 \pi$-exchange, contain two shorter-range contributions. In the last section we give the summary. 


\section{Reactions in the $3 \mathrm{~N}$ continuum}

All observables for elastic Nd scattering can be obtained from an amplitude $T|\phi\rangle$ which fulfills the $3 \mathrm{~N}$ Faddeev equation [14]

$$
\begin{aligned}
T|\phi\rangle & =t P|\phi\rangle+\left(1+t G_{0}\right) V_{4}^{(1)}(1+P)|\phi\rangle+t P G_{0} T|\phi\rangle \\
& +\left(1+t G_{0}\right) V_{4}^{(1)}(1+P) T|\phi\rangle .
\end{aligned}
$$

The initial channel state $|\phi\rangle$ is composed of a deuteron and a momentum eigenstate of the projectile nucleon. On top of $2 \mathrm{~N}$ forces with their off-the-energy shell t-matrix t for nucleons 2 and 3 , also a $3 \mathrm{NF}$ is included and $V_{4}^{(1)}$ is the part of it which is symmetrical under exchange of nucleons 2 and 3. The permutation operator $\mathrm{P}$ takes into account the identity of the nucleons and $G_{0}$ is the free $3 \mathrm{~N}$ propagator.

Using the realistic NN forces: AV18 [1], CD Bonn [2], Nijm1, Nijm2, and Nijm93 [3] one gets in general predictions for $3 \mathrm{~N}$ scattering observables which agree well with data at energies below $\approx 30 \mathrm{MeV}$. A fairly complete overview of those theoretical predictions in comparison to data is presented in [15, 16]. At higher energies discrepancies develop. They are exemplified for the elastic scattering cross section in the left panels of Figs.11 and 2. The large discrepancy in the minimum of the elastic scattering cross section obtained with $\mathrm{NN}$ forces only seen for energies above $\approx$ $60 \mathrm{MeV}$ is removed for energies below $\approx 140 \mathrm{MeV}$ when 3NF's are included which reproduce the experimental triton binding energy [17, 16] (see left panels of Figs. 1] and 2). A similar behavior shows up for the high energy deuteron vector analyzing power $A_{y}(d)[16,18,19,20]$. But there are many spin observables for which large 3NF effects are predicted and where the TM and the Urbana IX do not reproduce the data [16]. This is the case e.g. for the nucleon analyzing power $A_{y}$ [16, 21] and for the deuteron tensor analyzing powers [16]. In both cases the data cannot be reproduced by pure $2 \mathrm{~N}$ force predictions either. Therefore observables of elastic $\mathrm{Nd}$ scattering can be identified, which are sensitive to the $3 \mathrm{NF}$ structure. With precise data on such spin observables it should be possible to reveal the proper spin structure of the $3 \mathrm{NF}$.

\section{Relativistic effects in elastic Nd scattering}

There are large discrepancies at higher energies between data and theory in elastic Nd scattering which cannot be removed by adding standard 3NF's (see left panel of Fig.2). They require to study the magnitude of relativistic effects. We used an instant form relativistic approach which encompasses relativistic kinematics, boost corrections, and Wigner spin rotations [23, 24]. The boost effects turned out to be the most significant ones for the elastic scattering cross section at higher energies. They diminish the transition matrix elements at higher energies and lead, in spite of the increased relativistic phase-space factor as compared to the nonrelativistic one, to rather small effects in the cross section, mostly restricted to the backward angles [23] (see right panels of Figs.1 and 2). Higher energy elastic scattering spin observables are only slightly modified by relativity [23, 24]. 


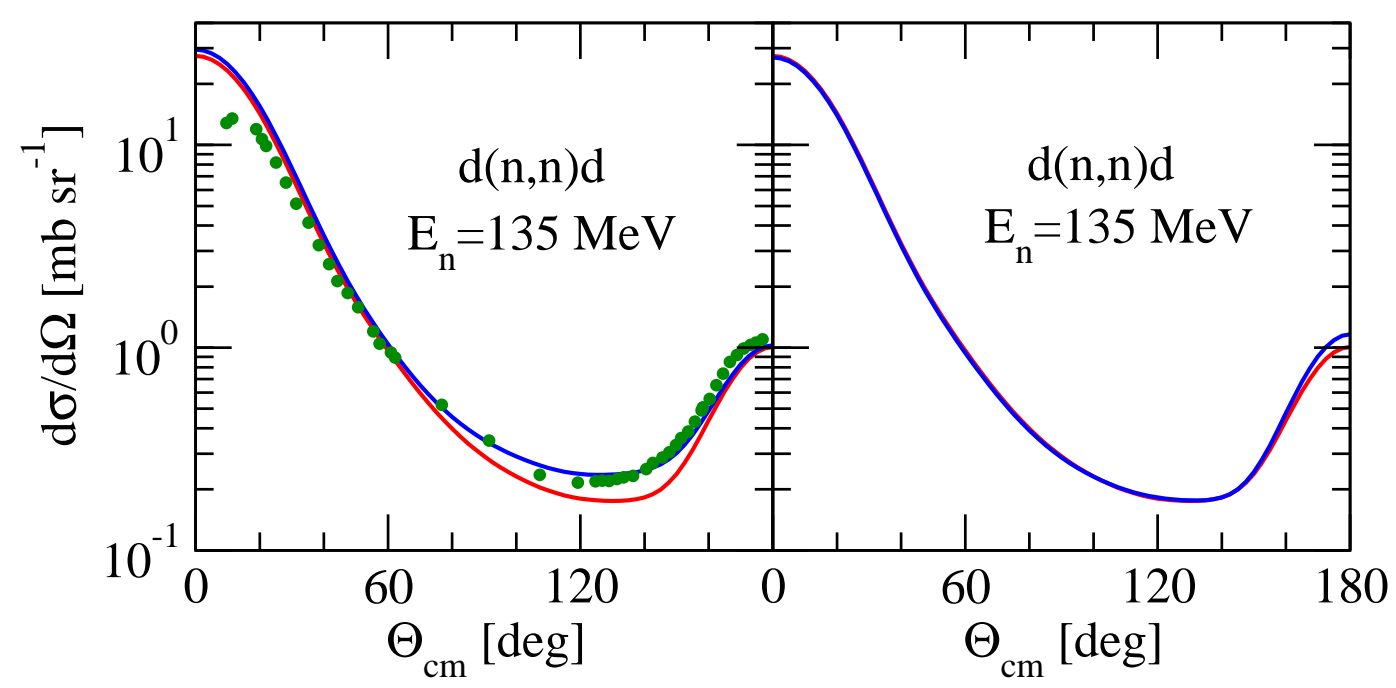

Figure 1: (Color online) The angular distribution for nd elastic scattering at neutron energy $E_{l a b}=135 \mathrm{MeV}$. The pd experimental data (solid dots) are from [18]. In the left and right panel the solid (red) line represents predictions of the CD Bonn potential alone. The solid (blue) line in the left panel shows the CD Bonn+TM99 $3 \mathrm{NF}$ predictions and in the right panel the results of relativistic calculations based on the CD Bonn potential.

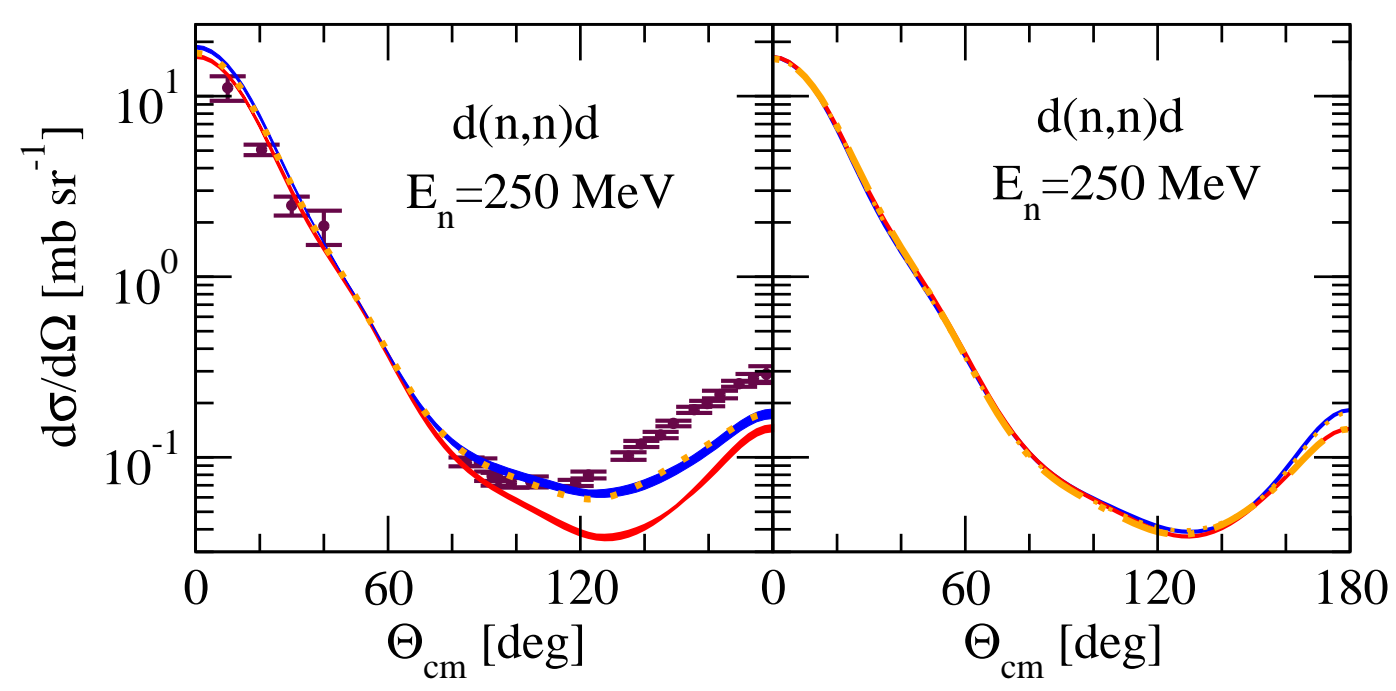

Figure 2: (Color online) The angular distribution for nd elastic scattering at neutron energy $E_{l a b}=250 \mathrm{MeV}$. The nd experimental data (solid dots) are from [22]. In the left panel the light shaded (red) and dark shaded (blue) bands result from predictions of (semi)phenomenological potentials alone (AV18, CD Bonn, Nijm1, Nijm2) and combined with TM99 3NF, respectively. The dotted (orange) line is the prediction of the AV18+TM99 3NF. In the right panel the solid (red) and dashed-dotted (orange) line (they are practically overlapping) is the prediction of the CD Bonn and AV18 potential, respectively. The solid (blue) and the dotted (orange) lines (also practically overlapping) are predictions of relativistic calculations based on the CD Bonn and AV18 potential, respectively. 


\begin{tabular}{|c|c|c|c|c|}
\hline Potential & LS cut-off $[\mathrm{MeV}]$ & SFR cut-off $[\mathrm{MeV}]$ & $E_{d}[\mathrm{MeV}]$ & $P_{d}[\%]$ \\
\hline N2LO 101 & 450 & 500 & -2.1922 & 3.536 \\
N2LO 102 & 600 & 500 & -2.1842 & 4.566 \\
N2LO 103 & 550 & 600 & -2.1887 & 4.383 \\
N2LO 104 & 450 & 700 & -2.2019 & 3.613 \\
N2LO 105 & 600 & 700 & -2.1997 & 4.709 \\
\hline N3LO 201 & 450 & 500 & -2.2161 & 2.727 \\
N3LO 202 & 600 & 600 & -2.2212 & 3.545 \\
N3LO 203 & 550 & 600 & -2.2193 & 3.283 \\
N3LO 204 & 450 & 700 & -2.2187 & 2.844 \\
N3LO 205 & 600 & 700 & -2.2232 & 3.634 \\
\hline
\end{tabular}

Table 1: The cut-off's for Lippmann-Schwinger equation (LS) regularization and spectral function regularization (SFR) used for different $\mathrm{N}^{2} \mathrm{LO}$ and $\mathrm{N}^{3} \mathrm{LO}$ potentials [10, 11] together with their deuteron properties.

\section{Results with chiral $\mathrm{NN}$ and $3 \mathrm{~N}$ forces}

The smallness of relativistic effects indicates that very probably the short range contributions to the $3 \mathrm{NF}$ are responsible for the higher energy elastic scattering discrepancies. Recently constructed $\mathrm{N}^{3} \mathrm{LO}$ chiral $\mathrm{NN}$ potentials extended the energy region with a highly improved description of the NN experimental phase-shifts up to about $\approx 150 \mathrm{MeV}$ [11]. It opened thus the way to apply chiral dynamics in analyses of higher energy elastic $\mathrm{Nd}$ scattering. At lower energies up to $\approx 65 \mathrm{MeV}$ chiral $\mathrm{N}^{2} \mathrm{LO} \mathrm{NN}$ and $3 \mathrm{~N}$ forces proved to be successfull [12].

We used 5 sets of $\mathrm{N}^{2} \mathrm{LO}$ and $\mathrm{N}^{3} \mathrm{LO} \mathrm{NN}$ forces [11] shown in Table 1 alone or combining them with 2 sets of $\mathrm{N}^{2} \mathrm{LO}$ chiral 3NF's [12], both with the same large-momentum cut-off parameter $\Lambda=500 \mathrm{MeV}$. Those two 3NF's, in the following denoted by 500A and 500B, differ in the values of the $\mathrm{D}$ and $\mathrm{E}$ parameters which appear in two short-range contributions to that $3 \mathrm{NF}$ [12]: in the $\pi$ exchange and $2 \mathrm{~N}$ contact term (D) and in the $3 \mathrm{~N}$ contact term (E). Their values are: $D=-1.1128$, $E=-0.6590$, and $D=8.1413, E=-2.0289$ for 500A and 500B, respectively.

The improvement of the NN phase-shift data description at higher energies going from $\mathrm{N}^{2} \mathrm{LO}$ to $\mathrm{N}^{3} \mathrm{LO} N \mathrm{~N}$ potentials is clearly visible in the reduced spread of $\mathrm{Nd}$ elastic scattering cross sections (see left and middle panel in Fig. 3). With increasing nucleon energy and with worsened description of the NN phase-shifts data by $\mathrm{N}^{3} \mathrm{LO} N \mathrm{~N}$ potentials also the band of $\mathrm{Nd}$ cross section predictions widens as exemplified in the right panel of Fig. 3 for $E=200 \mathrm{MeV}$. Thus an application of chiral potentials would require an even higher order in the expansion.

Despite the significant reduction of the spread seen at $135 \mathrm{MeV}$ some differences are left and a grouping of the $5 \mathrm{~N}^{3} \mathrm{LO} \mathrm{NN}$ potential predictions into 3 sets is visible in the middle panel of Fig. 3 . This can be traced back to different deuteron properties of those 5 potentials in comparison to the ones at $\mathrm{N}^{2} \mathrm{LO}$ (see Table1) and the significant change of the $l=0$ component of the deuteron wave function around $r \approx 2 \mathrm{fm}$ (low left panel of Fig. ॠ).

In Fig. 5 we exemplify that really the differences of the ${ }^{3} S_{1}-{ }^{3} D_{1} \mathrm{~N}^{3} \mathrm{LO} N \mathrm{~N}$ force components are responsible for the spreading of the $\mathrm{Nd}$ cross section predictions shown in the middle panel of 


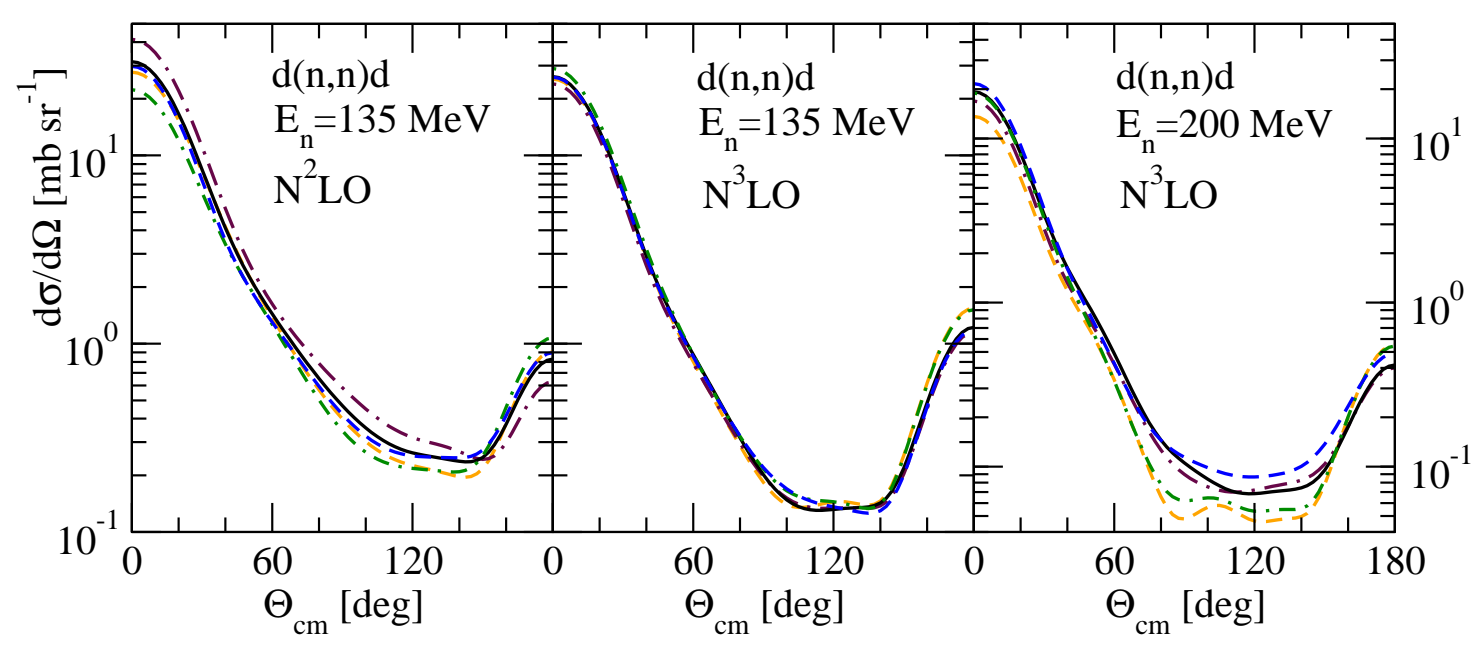

Figure 3: (Color online) The angular distribution for nd elastic scattering at neutron energy $E_{l a b}=135 \mathrm{MeV}$ (left and middle panels) and at $E_{l a b}=200 \mathrm{MeV}$ (right panel). In the left (middle and right) panel different lines are cross sections obtained with the chiral $\mathrm{N}^{2} \mathrm{LO}\left(\mathrm{N}^{3} \mathrm{LO}\right) \mathrm{NN}$ potentials from Table 11: dashed (orange) - $\mathrm{N}^{2}$ LO 101 ( $\mathrm{N}^{3}$ LO 201), dashed-dotted (maroon) - $\mathrm{N}^{2} \mathrm{LO} 102\left(\mathrm{~N}^{3} \mathrm{LO} 202\right)$, solid (black) $\mathrm{N}^{2} \mathrm{LO} 103\left(\mathrm{~N}^{3} \mathrm{LO}\right.$ 203), dashed-dotted (green) - $\mathrm{N}^{2}$ LO 104 ( $\left.\mathrm{N}^{3} \mathrm{LO} 204\right)$, and dashed (blue) - $\mathrm{N}^{2} \mathrm{LO} 105$ ( $\left.\mathrm{N}^{3} \mathrm{LO} 205\right)$.

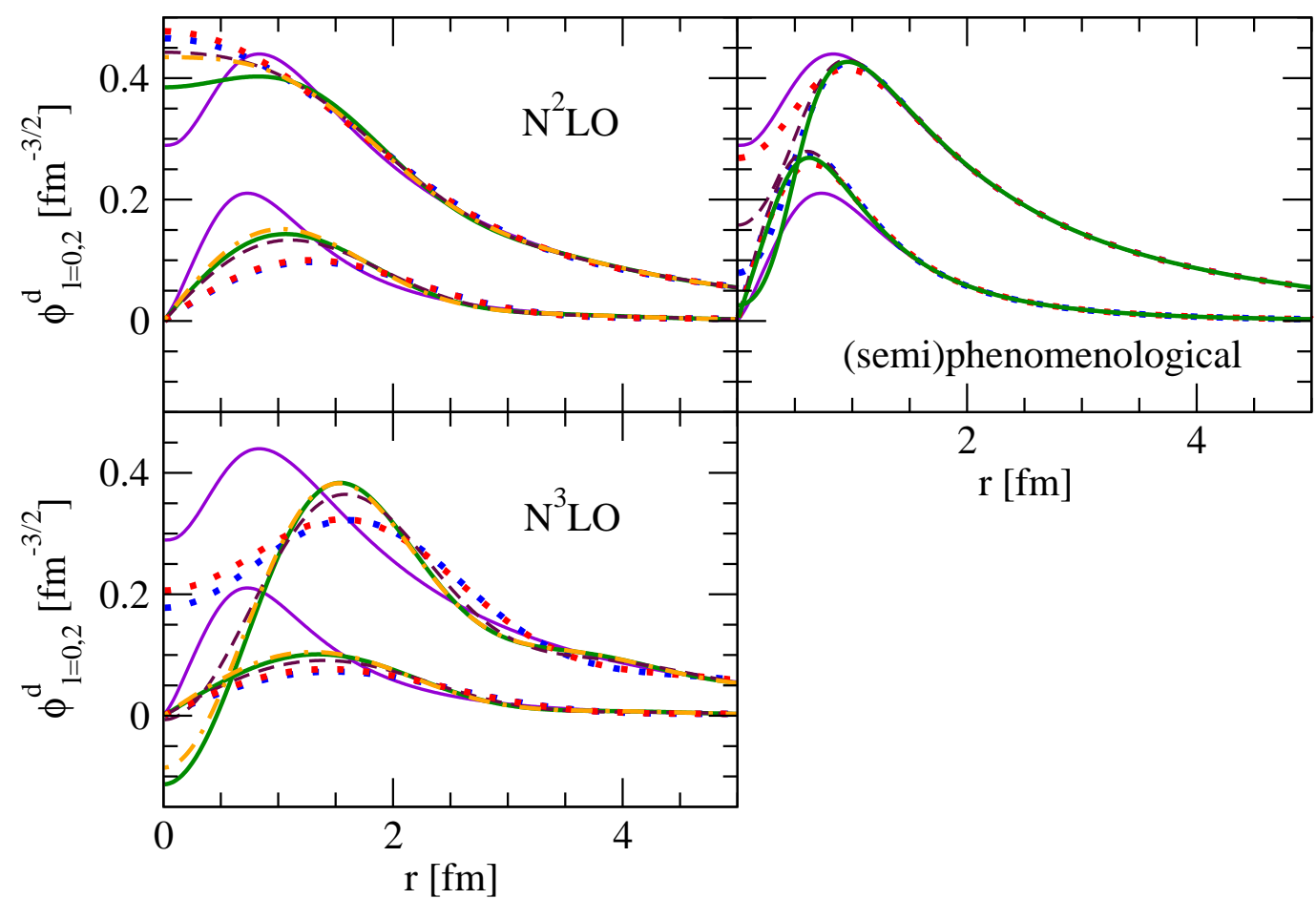

Figure 4: (Color online) The $l=0\left(\phi_{l=0}^{d}\right)$ and $l=2\left(\phi_{l=2}^{d}\right)$ deuteron wave functions for different NN potentials. In the right panel for (semi)phenomenological potentials: dotted (blue) - AV18, solid (violet) CD Bonn, dotted (red) - Nijm1, solid (green) - Nijm2, and dashed (maroon) - Nijm93. In the upper left (low left) panel for chiral $\mathrm{N}^{2} \mathrm{LO}\left(\mathrm{N}^{3} \mathrm{LO}\right)$ potentials from Table1: dotted (blue) - $\mathrm{N}^{2} \mathrm{LO} 101$ ( $\left.\mathrm{N}^{3} \mathrm{LO} 201\right)$, solid (green) - $\mathrm{N}^{2}$ LO 102 ( $\mathrm{N}^{3}$ LO 202), dashed (maroon) $\mathrm{N}^{2}$ LO 103 ( $\mathrm{N}^{3}$ LO 203), dotted (red) - N $\mathrm{N}^{2} \mathrm{LO} 104$ $\left(\mathrm{N}^{3} \mathrm{LO} 204\right)$, and dashed-dotted (orange) - $\mathrm{N}^{2} \mathrm{LO} 105\left(\mathrm{~N}^{3} \mathrm{LO} 205\right)$. In the left panels also CD Bonn results are shown by solid (violet) lines. 


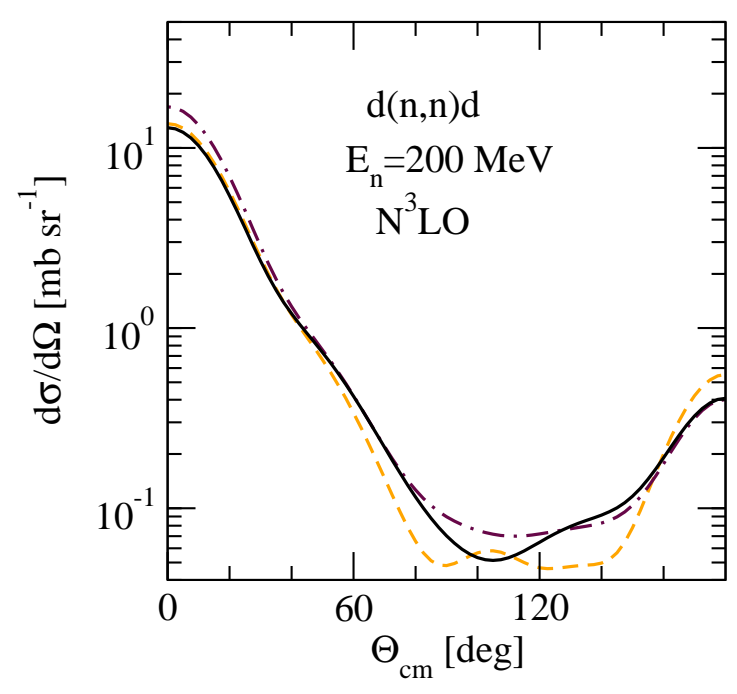

Figure 5: (Color online) The angular distribution for nd elastic scattering at neutron energy $E_{l a b}=200 \mathrm{MeV}$. The dashed (orange) (dashed-dotted (maroon)) line is prediction of the chiral $\mathrm{N}^{3} \mathrm{LO} 201$ (202) potential. The solid (black) line results when the ${ }^{3} S_{1}-{ }^{3} D_{1}$ force component of the $\mathrm{N}^{3} \mathrm{LO} 201$ is replaced by that of $\mathrm{N}^{3} \mathrm{LO}$ 202 while keeping all other components as in $\mathrm{N}^{3}$ LO 201.

Fig. 3. To this aim we present results with exchanged ${ }^{3} S_{1}-{ }^{3} D_{1}$ forces. It remains to be seen whether consistent $3 \mathrm{NF}^{\prime} \mathrm{s}$ at $\mathrm{N}^{3} \mathrm{LO}$ or currents in electromnagnetic processes on the deuteron will wash out those differences.

The significantly deeper minima for $\mathrm{N}^{3} \mathrm{LO} N \mathrm{~N}$ potentials (see the middle panel in Fig. 3) clearly require a corresponding larger impact of consistent $\mathrm{N}^{3} \mathrm{LO} 3 \mathrm{NF}$ 's which are not yet programmed. Using instead the inconsistent combination with the 500A or 500B $\mathrm{N}^{2} \mathrm{LO} 3 \mathrm{NF}$ 's sheds at least light on the importance of short range terms in the $3 \mathrm{NF}$. This is shown in Figs. 6 and 7, where the two different $\mathrm{N}^{2} \mathrm{LO}$ 3NF's lead to distinctly different results for higher energy spin observables. Moreover the description of the data is quite bad.

\section{Summary}

Solving $3 \mathrm{~N}$ scattering exactly in a numerical sense up to energies below the pion production threshold allows one to test the $3 \mathrm{~N}$ Hamiltonian based on modern NN potentials and 3NF's. At the higher energies for some observables large $3 \mathrm{NF}$ effects are predicted when using (semi)phenomenological models such as TM and Urbana IX. Some Nd elastic scattering cross sections and polarization data support these predictions. In some other cases, however, defects of the (semi)phenomenological 3NF's are demonstrated. Relativistic effects are found to be small for the elastic scattering cross section and negligible for higher energy spin-observables. The existing high energy discrepancies which remain even when Urbana IX or TM 3NF's are included indicate the importance of short-range contributions to a 3NF. Application of chiral NN interactions alone or combined with chiral $3 \mathrm{NF}$, which, in addition to a $2 \pi$-exchange contribution, comprises also two shorter-range terms, seems to support that indication.

It can be expected that a precise and rich data basis, comprising elastic scattering and breakup 


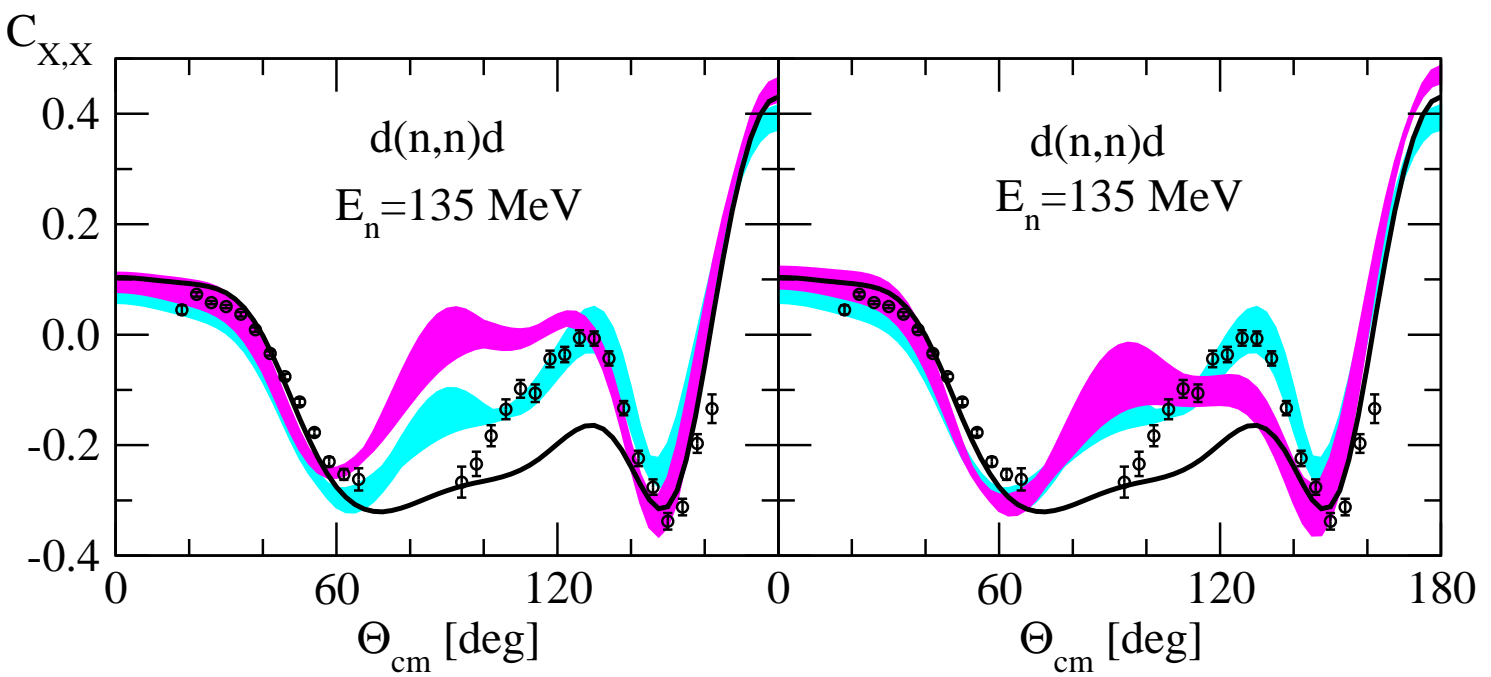

Figure 6: (Color online) The spin correlation coefficient $C_{x x}$ in nd elastic scattering at neutron energy $E_{l a b}=135 \mathrm{MeV}$. In the left and right panels the light shaded (cyan) band is based on predictions of chiral $\mathrm{N}^{3} \mathrm{LO}$ NN potentials from Table1. The dark shaded (magenta) band in the left and right panels result when chiral $\mathrm{N}^{3} \mathrm{LO}$ potentials are combined with chiral $\mathrm{N}^{2} \mathrm{LO}$ 3NF 500A and 500B, respectively. The solid (black) line is the prediction of the AV18+Urbana IX 3NF. The pd experimental data (open cirles) are from [20].

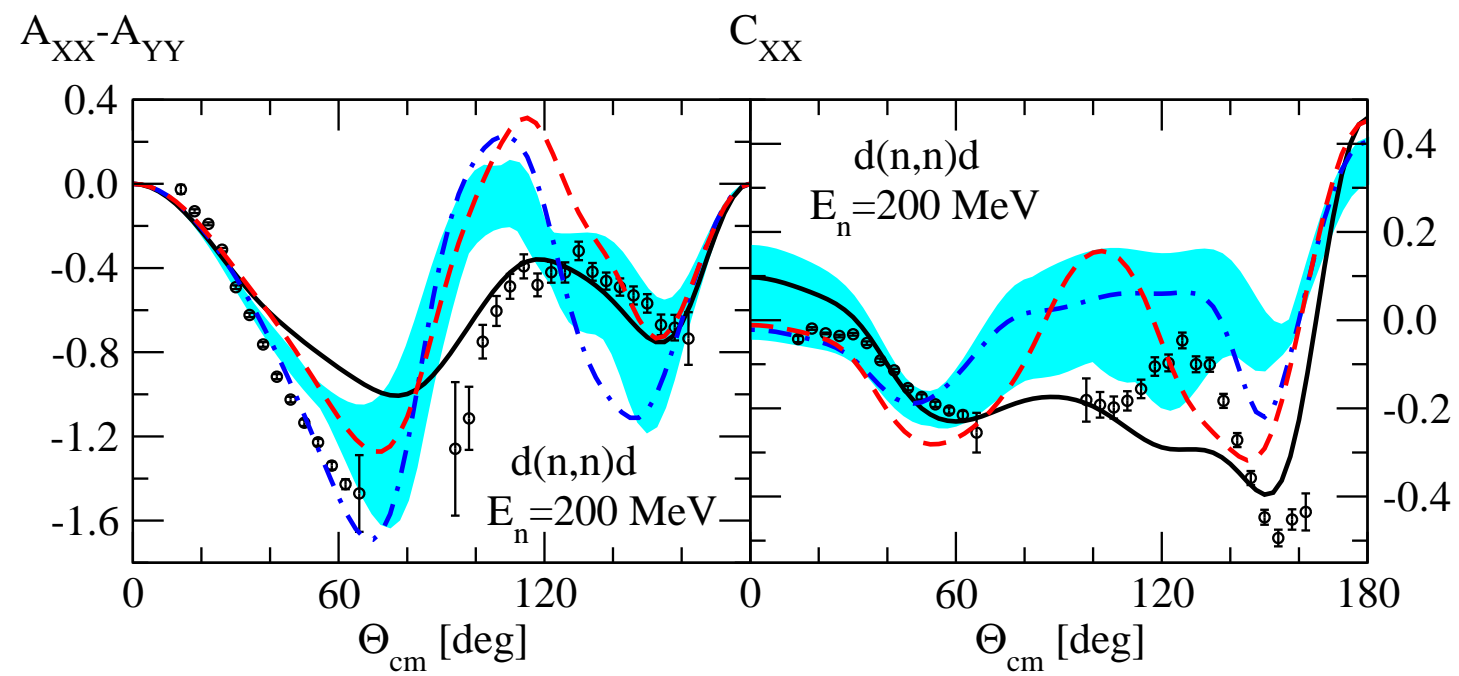

Figure 7: (Color online) The tensor analyzing power $A_{x x}-A_{y y}$ (left panel) and spin correlation coefficient $C_{x x}$ (right panel) in nd elastic scattering at neutron energy $E_{l a b}=200 \mathrm{MeV}$. The light shaded (cyan) band is based on predictions of chiral $\mathrm{N}^{3} \mathrm{LO}$ NN potentials from Table1. The dashed-dotted (blue) and dashed (red) lines result when chiral $\mathrm{N}^{3} \mathrm{LO} 201$ potential is combined with the chiral $\mathrm{N}^{2} \mathrm{LO} 3 \mathrm{NF} 500 \mathrm{~A}$ and 500B, respectively. The solid (black) line is the prediction of the AV18+Urbana IX 3NF. The pd experimental data (open cirles) are from [20]. 
data, will suffice to establish the proper spin structure of $3 \mathrm{~N}$ forces. Chiral perturbation theory providing consistent $\mathrm{NN}$ and $3 \mathrm{~N}$ forces will play an important role in these studies.

\section{References}

[1] R. B. Wiringa, V. G. J. Stoks, R. Schiavilla, Phys. Rev. C51, 38 (1995).

[2] R. Machleidt, Phys. Rev. C63, 024001 (2001).

[3] V. G. J. Stoks et al., Phys. Rev. C49, 2950 (1994).

[4] A. Nogga, H. Kamada, W. Glöckle, Phys. Rev. Lett. 85, 944 (2000).

[5] M. Viviani, Nucl. Phys. A631, 111c (1998).

[6] S. C. Pieper and R. B. Wiringa, Annu. Rev. Nucl. Part. Sci. C51, 53 (2001); S. C. Pieper, Nucl. Phys. A751, 516c (2005).

[7] J. Fujita, H. Miyazawa, Prog. Theor. Phys. 17, 360 (1957).

[8] B. S. Pudliner et al., Phys. Rev. C56, 1720 (1997).

[9] S. A. Coon et al., Nucl. Phys. A317, 242 (1979); S. A. Coon, W. Glöckle, Phys. Rev. C23, 1970 (1981).

[10] E. Epelbaum et al., Nucl. Phys. A747, 362 (2005).

[11] E. Epelbaum, Prog. Part. Nucl. Phys. 57, 654 (2006); E. Epelbaum et al., arXiv: 0811.1338v1 [nucl-th], to appear in Rev. Mod. Phys..

[12] E. Epelbaum et al., Phys. Rev. C66, 064001 (2002).

[13] P. Navratil et al., Phys. Rev. Lett. 99, 042501 (2007).

[14] D. Hüber et al., Acta Phys. Polonica B28, 167 (1997).

[15] W. Glöckle et al., Phys. Rep.274, 107 (1996).

[16] H. Witała et al., Phys. Rev. C63, 024007 (2001).

[17] H. Witała et al., Phys. Rev. Lett. 81, 1183 (1998).

[18] H. Sakai et al., Phys. Rev. Lett. 84, 5288 (2000).

[19] R. V. Cadman et al., Phys. Rev. Lett. 86, 967 (2001).

[20] B. v. Przewoski et al., Phys. Rev. C 74, 064003 (2006).

[21] R. Bieber et al., Phys. Rev. Lett. 84, 606 (2000).

[22] Y. Maeda et al., Phys. Rev. C 76, 014004 (2007).

[23] H. Witała et al., Phys. Rev. C 71, 054001 (2005).

[24] H. Witała et al., Phys. Rev. C 77, 034004 (2008). 